\title{
Multiple regions of the murine coronavirus spike glycoprotein influence neurovirulence
}

\author{
Joanna J Phillips, MingMing Chua, Su-hun Seo, and Susan R Weiss \\ Department of Microbiology, University of Pennsylvania School of Medicine, Philadelphia, Pennsylvania, USA
}

\begin{abstract}
The spike (S) glycoprotein of mouse hepatitis virus (MHV) is a major determinant of neurovirulence. Using targeted recombination we previously demonstrated that the $S$ gene of the highly neurovirulent MHV-4 conferred a dramatic increase in neurovirulence to the mildly neurovirulent MHV-A59. To identify the genetic determinants of neurovirulence within the MHV-4 spike, we generated isogenic recombinant viruses containing various MFV-4MFrV-A59 chimeric spike genes, and studied their phenotypes in vivo. The MHV-4/MHVA59 chimeric spike genes consisted of either reciprocal exchanges between the S1 and S2 spike subunits, or smaller exchanges specifically in the hypervariable region (HVR) of S1. The chimeric spike gene containing recombinants all exhibited efficient replication in vitro, yet many were severely attenuated for virulence in vivo. Furthermore, these attenuated recombinants exhibited decreased titers of infectious virus in the brain relative to the parental recombinant viruses containing the full-length MHV-4 or MHV-A59 spike genes. This is the first report that compares the neurovirulence and pathogenesis of isogenic viruses with defined alterations in the MHV spike protein. From these studies, it appears that the interactions of multiple regions of the MHV spike, including the HVR, act in concert to allow for efficient infection of and virulence in the murine central nervous system. Journal of NeuroVirology (2001) 7, 421-431.
\end{abstract}

Keywords: mouse hepatitis virus; encephalitis; recombinant coronaviruses; neurovirulence

\section{Introduction}

Murine coronaviruses, or mouse hepatitis viruses (MHV), are enveloped positive-stranded RNA viruses that can induce a variety of respiratory, gastrointestinal, and neurologic diseases in rodents. As with a number of viral pathogens, the severity and organ tropism of the disease depend, in part, on the viral strain. Infection with the highly neurovirulent MHV type 4 (MHV-4, a strain JHM isolate) results in a potentially fatal acute encephalomyelitis (Dalziel et al, 1986). Infection with a similar dose of the mildly neurovirulent MHV type A59 (MHV-A59) results in a'much less severe disease with only mild

Address correspondence to Susan R Weiss, Department of Mierobiology, University of Pennsylvania School of Medicine, 203A fohnson Pavillion, 36th Street and Hamilton Walk, Philadelphia, BA 19104-6076. USA.E-mail: Weisssr@mail.med.upenn.edu

Received 16 February 2001; revised 17 April 2001; accepted 26 April 2001. encephalomyelitis and virtually no mortality (Lavi et al, 1986; Lavi et al, 1988). Recently, using targeted recombination, we have demonstrated that the spike gene of MHV-4 is sufficient to confer this highly neurovirulent phenotype to MHV-A59 (Phillips et al, 1999).

The MHV spike or S glycoprotein, expressed on the virion envelope and on the plasma membrane of infected cells, plays a vital role in viral entry, viral spread, and in the immune response to infection (Collins et al, 1982; Buchmeier et al, 1984; Dveksler et al, 1991; Castro and Perlman, 1995; Bergmann et al, 1996). Depending both on the viral strain and the host cell the spike is posttranslationally cleaved dividing the spike into an amino-terminal, $S 1$, subunit and a carboxy-terminal, S2, subunit (Sturman et al, 1990). S1, thought to form the globular head of the spike, mediates binding to the viral receptors (Collins et al, 1982; DeGroot et al, 1987; Kubo et al, 1994) that are members of the CEACAM (previously Bgp (Beauchemin et al, 1999)) subclass of the 
large carcinoembryonic anligen (C, I: $\Lambda$ ) gene family (Dveksler et al, 1991; Williams et al, 1991). Subsequent to receptor-binding, there is thought to be a conformational change in spike which allows S2, the stalk portion of the peplomer. 10 induce viruscell membrane fusion (Collins el al, 1982; DeGroot et al, 1987; Sturman et al, 1990; Weismiller et al, 1990). Subsequent to infection. S expressed on the host cell membrane can also mediate cell-cell fusion. During an in vivo infection, the MHV spike is also an important target for bolh humoral and cellular immune responses (Collins ef al, 1982; Fleming et al, 1983; Castro and Perlman, 1995; 13ergmann et al, 1996).

Despite the dramatic differences in neurovirulence conferred by spike proteins of MHV-4 and MHVA59 they are highly homologous proteins, sharing $95 \%$ amino acid identity in S2 and $90 \%$ identity in S1. Within the S1 subunit of the less neurovirulent MHV-A59 spike, however, there is deletion of 52 amino acids in a region termed the hypervariable region (HVR) (Luytjes et al, 1987). The spike HVR, loosely defined between amino acids 400 and 600 , exhibits a great deal of variation in length from strain to strain (Parker et al, 1989; Banner et al, 1990). The highly neurovirulent MHV-4 has the longest known HVR, but deletions in this region of up to 159 amino acids have been reported (Parker et al, 1989). The specific role of the IHVR in pathogenesis appears to be complex and multifunctional. Deletions or mutations in this region have been found to alter viral fusion, eliminate neutralizing monoclonal antibody epitopes, and abrogate $\mathrm{CD}^{+}{ }^{+}$T-cell epitopes (Dalziel ot al, 1986; Fleming et al, 1986; Wege et al, 1988; Parker et al, 1989; Taguchi and Fleming, 1989; Gallagher et al, 1990; Pewe et al, 1996; Tsai et al, 1999; Wang et al, 1992). In addition, deletions or mutations in the HVR have been associated with neuroattenuation. In one such study, a 142-amino acid deletion in the HVR was associated with decreased neurovirulence and decreased viral spread in the CNS (Fazakerley et al, 1.992).

With the recent development of targeted RNA recombination the struclural genes of MHV have become accessible, for the first time, to genetic manipulation (Fischer et al, 1997; Leparc-Goffart et al, 1998). Using this technique we have definitively demonstrated that the $\mathrm{S}$ gene is a major determinant of $\mathrm{MHV}$ neurovirulence (Phillips et al, 1999). "To further identify genetic determinants of neurovirulence within the MHV spike, we generated a series of recombinants containing MHV-4/MHV- $A 59$ chimeric spike genes, and examined the pathogenesis of these isogenic viruses that differed exclusively in spike. From these studies, we conclude that multiple regions of the MHV-4 spike, including the hypervariable region, are required for efficient infection and virulence in the mouse CNS.

\section{Results}

Pathogenesis of recombinants expressing S1/S2 chimeric spike genes

To begin to identify specific regions of the MHY spike which play a role in neurovirulence, we generated recombinant viruses containing exchanges between the S1 and S2 subunits of the highly neurovirulent MHV-4 spike and the much less neurovirulent MHVA59 spike. The recombinants were generated using a modification of the targeted recombination strategy as recently described by Kuo et al (2000) (see Materials and methods). To reduce the risk of secondary site mutations influencing the viral phenotype, two independently selected recombinants (R), as indicated by their numbering, were isolated and characterized for each desired recombinant virus. As shown in Figure 1, the recombinants containing the S1 of MFV4 and the S2 of MHV-A59 were named $S 1_{4} R 70$ and $S 1_{4} \mathrm{R} 71$, and the recombinants containing the $\mathrm{S} 1$ of MHV-A59 and the S2 of MHV-4 were called S2 ${ }_{4}$ R81 and $S 2{ }_{4} R 82$. The S1/S2 junction was located just $3^{\prime}$ to the cleavage site at amino acid 775 (see Materials and

\begin{tabular}{|c|c|c|}
\hline Spike & Virus & $\left(L_{50}\right)$ \\
\hline & $\mathrm{S}_{4} \mathrm{R} 29$ & 0.3 \\
\hline 775 & $\begin{array}{r}S 1{ }_{4} \mathrm{R} 70 \\
\mathrm{R} 71\end{array}$ & $\begin{array}{l}>4.7 \\
>4.7\end{array}$ \\
\hline $\begin{array}{r} \\
775\end{array}$ & $\begin{array}{c}\mathrm{S} 2{ }_{4} \mathrm{R} 81 \\
\mathrm{R} 82\end{array}$ & $\begin{array}{l}>4.5 \\
>3.5\end{array}$ \\
\hline $434-575$ & $\begin{array}{r}\mathrm{S}_{4} \Delta \mathrm{HVR} 160 \\
\mathrm{R} 161\end{array}$ & $\begin{array}{l}>4.1 \\
>4.3\end{array}$ \\
\hline $488-600$ & $\begin{array}{r}\mathrm{S}_{4} \mathrm{HV}-\mathrm{A} 59 \mathrm{R} 131 \\
\mathrm{R} 133\end{array}$ & $\begin{array}{r}4.5 \\
>4.4\end{array}$ \\
\hline$\frac{1}{412-614}$ & $\begin{array}{r}\mathrm{S}_{\mathrm{A} 59} \mathrm{HV}-4 \mathrm{R} 51 \\
\mathrm{R} 52\end{array}$ & $\begin{array}{l}3.4 \\
3.2\end{array}$ \\
\hline 5.74 - & $\mathrm{S}_{\mathrm{A} 59} \mathrm{R} 16$ & 3.7 \\
\hline
\end{tabular}

Figure J Schematic: diagram of the spike gene from the recombinant viruses and their corresponding virulence following intracranial inoculation. $L D_{50}$ assays were conducted as described previously (Hingley of al, 1994). The recombinant viruses are represented as inclicaled. $S_{A 59} R 16$ and $S_{i} R 29$ contain the MIIV- $\Lambda 59$ spike and the MHV-4 spike, respectively. $S 1_{4} R 70$ and 71 contain the S1 of the MHV-4 spike with the S2 of the MHV-A59 spike, and $S 2$ R81 and 82 contains the S1 of MHV-A59 and the S2 of MHV- 4 $S_{i} \triangle F V R 160$ and 61 represent recombinants that have a 142 -amino acid deletion in the MHV-4 hypervariable region (HVR), while $\mathrm{S}_{4} \mathrm{HV}-\mathrm{A}$ 59R131 and R133 contain an exchange of the MHV-4 HVR for the corresponding MLHV-A59 region. $\mathrm{S}_{\mathrm{A} 59} \mathrm{HV}-4 \mathrm{R} 51$ and $\mathrm{R} 52$ contain the MHV-A5t] spike with the HVR of MHV-4. The amino acid residues at the exchange sites are indicated by their position in the MHV-4 spike. Recombinant S2 $2_{4} 81$ contained a single coding mutation L661Q, and recombinants $S_{4} H V-A 59 R 131$ and R133 each contained a single coding mutation $S 3591$ and N374Y, respectively. Groy bars imdicala the MIVV- $\Lambda 59$ sequence, and closed bars indicate the MIV-4 sequence. The 52-amino acid deletion in the MHV-A59 IIVR is indicated. 
Methods). As controls, recombinants containing the full-length spike gene of MHV-4, $\mathrm{S}_{4} \mathrm{R} 29$, or MHV-A59, $S_{A 59} R 16$, were isolated and characterized in parallel. To further decrease the impact of secondary site mutations in the recombinant viruses, we sequenced the entire $S$ gene from at least one of each pair of recombinants. $S_{4} R 29, S_{A 59} R 16$, and $S 1_{4} R 71$ contained no secondary site mutations in spike. $S 2_{4} \mathrm{R} 81$ contained a single point mutation in the $S 1$ subunit that resulted in a codon change L661Q. An independently isolated recombinant virus, $\mathrm{S}_{4} \mathrm{R} 82$, contained no secondary site mutations in $\mathrm{S}$.

To examine the efficiency of replication of the chimeric spike gene recombinants we performed growth curves on L2 cell monolayers with $S 1_{4} R 70$, $S 2_{4} R 81, S_{4} R 29$, and $S_{A 59} R 16$. The results are shown in Figure 2A. Consistent with previous reports, recombinants containing the MHV-A59 spike, $\mathrm{S}_{\mathrm{A} 59} \mathrm{R}$, replicated to higher titer in cell culture than recombinants containing the MHV-4 spike, $\mathrm{S}_{4} \mathrm{R}$ (Phillips et al, 1999). Both chimeric spike gene recombinants, $S 1_{4} \mathrm{R} 70$ and $\mathrm{S} 2{ }_{4} \mathrm{R} 81$, replicated in cell culture, and exhibited similar kinetics as the two parental recombinants, $S_{A 59} R$ and $S_{4} R$. The titers of infectious virus, however, were intermediate between $S_{A 58} R$ and $S_{4} R$.

The plaque morphology of the chimeric spike gene recombinants was also intermediate between $S_{A 59} R$ and $\mathrm{S}_{4} \mathrm{R}$. At $48 \mathrm{~h}$ after infection of $\mathrm{L} 2$ cell monolayers, the $\mathrm{S}_{4}$ recombinants and the $\mathrm{S}_{2}$ recombinants exhibited a medium-plaque phenotype $(0.75$ $\mathrm{mm}$ and $0.5 \mathrm{~mm}$, respectively) as compared to the large-plaque phenotype produced by $S_{A 59} R(1.0 \mathrm{~mm})$ and the small-plaque phenotype produced by $S_{4} R$ $(0.25 \mathrm{~mm})$. Thus, the S1/S2 chimeric spike gene recombinants replicated efficiently in cell culture, but exhibited a slightly different in vitro phenotype from either of the parental MHV-4 or MHV-A59 spike containing recombinants.
A

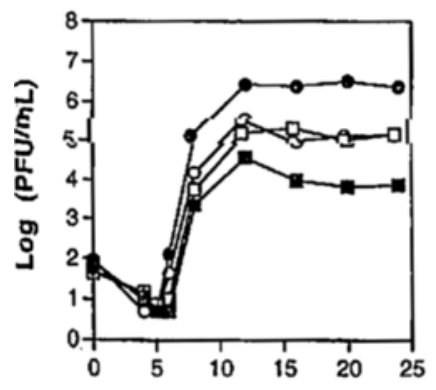

B

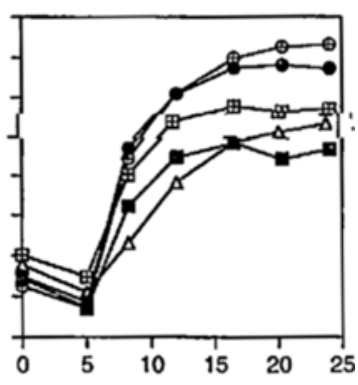

Hours Post Infection

Figure 2 Time course of recombinant virus production on $L 2$ cell monolayers. Each point represents the mean titer of duplicate samples. (A) Cells were infected at a multiplicity of infection of 1.8 PFU/cell with $S_{A s 9} R 16(\bullet), S 1_{4} R 70(\square), S 2{ }_{4} R 81(O)$, and $S_{4} R 29$ (i). (B) Cells were infected at a multiplicity of $0.4 \mathrm{PFU} / \mathrm{cell}$ with $S_{A 59} R 16(0), S_{A 59} H V-4 R 51$ (cross in O), $S_{4}$ HV-A59R131 (cross in G), $S_{4} \Delta H V R 160(\Delta)$, and $S_{4} R 29($ in).
To determine if either the S1 or the S2 subunit of the MHV-4 spike was sufficient to confer an increase in neurovirulence, we infected mice intracranially with $S 1_{4} \mathrm{R} 70, \mathrm{~S} 1_{4} \mathrm{R} 71, \mathrm{~S} 2{ }_{4} \mathrm{R} 81, \mathrm{~S} 2{ }_{4} \mathrm{R} 82, \mathrm{~S}{ }_{4} \mathrm{R} 29$, and $\mathrm{S}_{\mathrm{A} 59} \mathrm{R} 16$, monitored for lethality, and calculated $\mathrm{ZD}_{50}$ values. The neurovirulence of each recombinant is shown in Figure 1. The $L D_{50}$ for the $S_{4}$ and $S_{A 59}$ recombinants were similar to those reported previously (Phillips et al, 1999). The virulence of the chimeric $S$ gene recombinants, however, was very different from either of the parental viruses. Both $\mathrm{S}_{4}$ and $S 2_{4}$ recombinants were highly attenuated for virulence. Consistent with this decrease in virulence, mice exhibited clinical signs such as hunched posture, ruffled fur, and abnormal gait only at high doses of virus ( $>1000$ PFU).

To determine if the reduced virulence of the chimeric spike gene recombinants was attributable to decreased viral replication in the brain, we titered brain homogenates for infectious virus at various days after intracranial inoculation with $10 \mathrm{PFU}$ of virus (see Materials and Methods). The results are shown in Figure 3. As we have shown previously, the titers of infectious virus in the brain were similar following inoculation with $S_{4} R$ and $S_{A 59} R$ despite the dramatic difference in virulence exhibited by the two recombinant viruses (Phillips et al, 1999). The two chimeric $S$ gene recombinants, however, exhibited markedly reduced virus titers. $S 1_{4} \mathrm{R} 70$ exhibited low levels of virus while the amount of infectious $\mathrm{S} 2{ }_{4} \mathrm{R} 81$ virus was at or below the level of detection (200 PFU/g). Thus, despite the high amino acid identity between the MHV-4 and MHV. A59 spike proteins they could not substitute functionally for one another in an in vivo infection. This

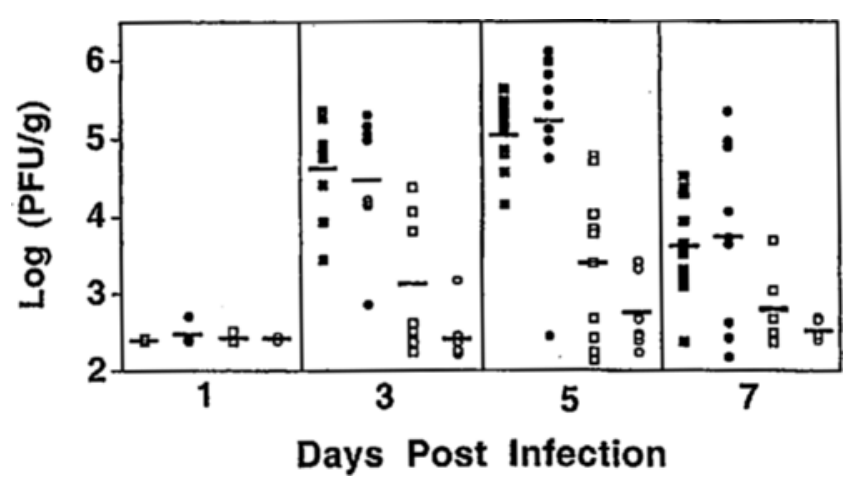

Figure 3 Titers of infectious virus in the brains of animals inoculated with viral recombinants containing chimeric MHV-4/MHVA59 $S$ gemes. Weanling $B 6$ mice were inoculated intracranially with 10 PFU of $S_{4} R 29(E), S_{A 59} R 16(\bullet), S 1_{4} R 70$ (D), or $S 2_{4} R 81(O)$. Animals were sacrificed at the indicated times, and virus titers in the brain were determined by plaque assay. The titers of infectious virus in the brain are given for each mouse and the mean virus titers are shown as bars ( $n=4-10$ mice per time point, data for days 5 and 7 are from three independent experiments). On day 5 p.i., the mean virus titers in $\mathrm{S}_{4} \mathrm{R} 29$-infected mice was significantly greater than in $S 2_{4} \mathrm{R} 81$-infected mice (two-tailed $t$-test, $P>0.0001$ ). The limit of detection was 200 PFU/g of tissue. 
result suggests that the high neurovirulence conferred by the MHV-4 spike protein requires specific homotypic interactions between regions in $\mathrm{S} 1$ and $\mathrm{S} 2$, and that disruption of these interactions has a major impact on the pathogenesis of the virus,

\section{Role of the HVR in pathogenesis}

Within the S1 subunit there is a region, termed the hypervariable region (HVR), which can tolerate extraordinary variation particularly with respect to its length (Banner et al, 1990; Parker et al, 1989). It has been proposed that the HVR has a vital function in MHV pathogenesis. Numerous variant viruses have been identified in which mutations or deletions in this region have been associated with alterations in neuropathogenesis (Dalziel et al, 1986; Fleming et al, 1986; Wege et al, 1988; Parker et al, 1989; Taguchi and Fleming, 1989; Gallagher et al, 1990; Wang et al, 1992). To definitively assess the role of the hypervariable region (HVR) in neurovirulence, we used targeted recombination to generate a series of recombinant viruses with small exchanges in the HVR between MHV-4 and MHV-A59. To determine if the HVR of the highly neurovirulent MHV-4 spike was necessary to confer an increase in neurovirulence, we generated recombinants in which the MHV-4 HVR was deleted or replaced with the MHV-A59 HVR (see Figure 1). Deletion of the MHV-4 HVR was based on a previously identified neutralizing monoclonal antibody escape mutant with a deletion in the HVR, and the two independently isolated recombinants containing this 142amino acid deletion were named $\mathrm{S}_{4} \triangle \mathrm{HVR} 160$ and $S_{4} \Delta$ HVR161 (Dalziel et al, 1986; Parker et al, 1989). Recombinants $\mathrm{S}_{4} \mathrm{HV}-\mathrm{A} 59 \mathrm{R} 131$ and $\mathrm{S}_{4} \mathrm{HV}-\mathrm{A} 59 \mathrm{R} 133$ contained the MHV-4 $S$ gene with replacement of a 112-amino acid region in the HVR with the corresponding 60-amino acid region from the MHV-A59 $S$ gene. In addition, to examine if the MHV-4 HVR was sufficient to confer some degree of increased neurovirulence we replaced the. MHV-A59 HVR with the MHV-4 HVR (see Figure 1). Thus, recombinants $\mathrm{S}_{\mathrm{A59}} \mathrm{HV}-4 \mathrm{R} 51$ and $\mathrm{S}_{\mathrm{A59}} \mathrm{HV}-4 \mathrm{R} 52$ contained the MHVA59 spike with replacement of a 150-amino acid region in the HVR with the corresponding 202-amino acid region from the MHV-4 spike. Once again, we sequenced the entire $S$ gene from at least one recombinant of each pair of recombinants. Recombinants $S_{4} \triangle H V R 160$ and $S_{A 59} H V-4 R 51$ contained no secondary site mutations. Sequencing of recombinant $\mathrm{S}_{4} \mathrm{HV}$-A59R131, containing the MHV-4 spike with. the MHV-A59 spike HVR, revealed a single coding mutation S359I. Interestingly we were unable to select a $\mathrm{S}_{4} \mathrm{HV}-\mathrm{A}_{5}$ 9R recombinant without a secondary site mutation. $\mathrm{S}_{4} \mathrm{HV}-\mathrm{A} 59 \mathrm{R} 133$, an independently isolated recombinant, contained a single coding mutation N374Y, and two additional recombinants, $\mathrm{S}_{4} \mathrm{HV}$-A59R132 and R134, were partially sequenced and found to contain single coding mutations (L1114F and L660Q, respectively). These two recombinants were not further characterized. The inability to select $\mathrm{S}_{4} \mathrm{HV}$-A59 recombinants without secondary site mutations in $\mathrm{S}$ suggests that these mutations were selected for during replication in cell culture.

The replication efficiency of these recombinants on L2 cell monolayers is shown in Figure 2B. The recombinants all exhibited similar kinetics of viral replication; however, the extent to which they replicated differed. In general, the growth phenotype of the recombinants segregated with the spike gene. Recombinants with primarily MHV-4 spike sequence, $\mathrm{S}_{4} \triangle \mathrm{HVR}$ and $\mathrm{S}_{4} \mathrm{HV}-\mathrm{A} 59 \mathrm{R}$, replicated to a similar extent as $\mathrm{S}_{4} \mathrm{R}$, and the HVR recombinant with primarily MHV-A59 spike sequence, $S_{A 59} H V-4 R$, replicated to a similar extent as $S_{A 59} R$. The plaque morphology of the recombinants was also dependent on the spike gene. $48 \mathrm{~h}$ p.i. of L2 cell monolayers, $\mathrm{S}_{\mathrm{A} 59} \mathrm{R}$, and $\mathrm{S}_{\mathrm{A59}} \mathrm{HV}-4 \mathrm{R}$ exhibited a large-plaque morphology $(1.0 \mathrm{~mm})$. The recombinants with primarily the MHV-4 spike exhibited smaller plaque-phenotypes. $\mathrm{S}_{4} \mathrm{HV}-\mathrm{A} 59 \mathrm{R}$ exhibited an intermediate-plaque size $(0.75 \mathrm{~mm})$, and $S_{4} \Delta$ HVR exhibited a delayed plaquephenotype as plaques were not yet visible at $48 \mathrm{~h}$ p.i. The delay in plaque-formation observed with $\mathrm{S}_{4} \Delta \mathrm{HVR}$ was consistent with previous reports associating deletions in the MHV-4 HVR with delayed fusion and decreased cytopathicity (Gallagher et al, 1990). In general, recombinants with alterations in the hypervariable region exhibited similar properties in cell culture as the parental spike from which they were derived.

To determine the effect of mutations in the HVR on virulence, we inoculated animals intracranially with serial dilutions of the recombinant viruses, observed them for lethality, and then calculated $L D_{50}$ doses. The results are shown in Figure 1. Removal of the MHV-4 HVR, either by deletion, $S_{4} \Delta H V R$, or by replacement with the MHV-A59 HVR, $\mathrm{S}_{4} \mathrm{HV}$ $A 59 R$, resulted in recombinant viruses with attenuated virulence. Thus the MHV-4 HVR was necessary for the highly neurovirulent phenotype conferred by the MHV-4 spike. In contrast, insertion of the MHV-4 HVR into the MHV-A59 spike, $S_{\text {A59 }} H V-4$, did not alter the virulence of the recombinant viruses indicating that the MHV- 4 HVR was not sufficient to confer an increase in virulence to the MHV-A59 spike. In addition, the similar virulence exhibited by $\mathrm{S}_{\mathrm{A} 59} \mathrm{R} 16$ and the two recombinants $S_{A 59} H V-4 R 51$ and $R 52$ indicate that an insertion of 52 amino acids is well tolerated in the MHV-A59 HVR.

To determine the effect of alterations in the HVR on the efficiency of viral replication in the brain, we inoculated mice intracranially with the HVR recombinants, and determined the titers of infectious virus in the brain at various times p.i. (Figure 4). Consistent with their poor virulence, the MHV-4 S gene containing recombinants with deletions or exchanges in the HVR exhibited dramatically reduced virus titers in the brain which were at or below the level of 


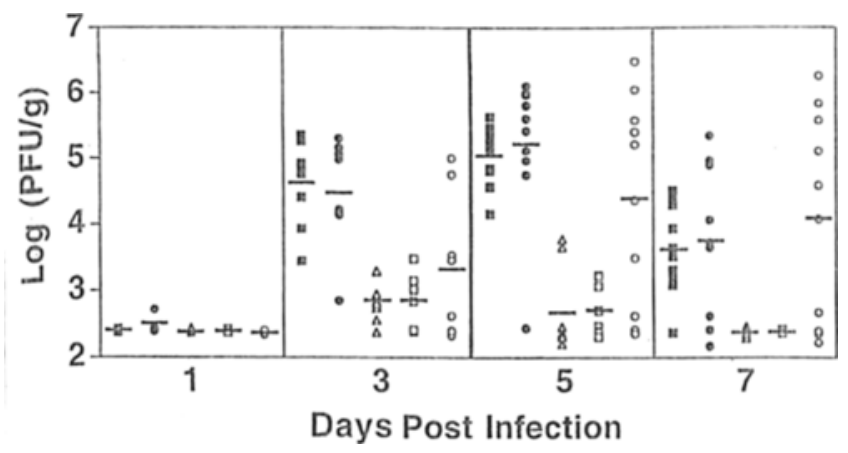

Figure 4 Titers of infortious virus in the brains of animals inoculated with recombinant viruses with altemtions in the llVR. As described in Figure 3, animals were inoculated int actanially

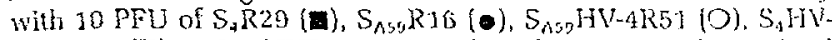
A59R131 (D), or $S_{4} \Delta$ HVR160 $(\Delta)$, and viral titers were determined at various days post infection. Each data point represents the titer of infectious virus from a single mouse and the mean virus liters are shown as bars $(n=4-10$ mice per time point, data for days 5 and 7 axe from three independent experiments). "The dila for the two parental recombinant viruses, $S_{4} R 29$ and $S_{A 59} R 16 i$, is the same as in Figure 3, and is shown here for comparison. 'llye linnt of detection was $200 \mathrm{PFU} / \mathrm{g}$ of tissue. On day $5 \mathrm{p}$.i., the mean titors of infectious virus in $S_{1} R 29$ infected mice was significantly gyroater than in $S_{i}$ HV-A59R131- or $S_{i} O H V R 160$-infecteri mice (two-lailer i-test, $P>0.0001$ (or both).

detection (200 PrU/g). On days 3 and 5 p.i. with $\mathrm{S}_{4} \triangle \mathrm{HVR}$ or $\mathrm{S}_{4} \mathrm{ITV}-\mathrm{A} 59 \mathrm{R}$ the titers of infectious virus were significantly different from the titers following infection with $S_{4} R 29$ (two-tailed $t$-test, $P>0.0001$ ). In contrast, replacement of the MHV-A59 HVR with the corresponding region from MFIV-4 did not resuit in a dramatic reduction in the amount of infectious virus recovered from the brain. Although $\mathrm{S}_{\mathrm{A} 59} \mathrm{HV}-4 \mathrm{R}$ exhibited similar average titers of infectious virus as $S_{n 59} R$, there was a large degree of animal to inimal variation as indicated in Figure 4 . On day 7 p.i., some animals exhibited relatively high titers of infectious virus. To demonstrate that these high titers late in infection were not attributable to the replication of variant viruses with secondary site mutations in the spike, we amplified virus from the brain at 7 days p.i., and sequenced the spike gene to generate a conisensus sequence. No secondary site mutations were found in the spike gene.

\section{Fusion phenotype of the recombinant viruses}

Despite efficient replication and growth in cell culture most of the chimeric spike containing recombinant viruses exhibited attenuated virulence in vivo. Due to the numerous selective pressures present during an in vivo infection it is possible that small alterations in viral entry, spread, or replication, which were not readily observable in cell culture, had a profound impact on virulence. Previous studies have found an association between alterations in the ability of the MHV spike to induce fusion and alterations in pathogenesis (Frana et al, 1985; Gallagher el al, 1990; Gallagher et al, 1991; Gallagher et al, 1992;
Rao and Gallighor, 1998). To examine lho fusion phenotype of the recombinant viruses following virus infection wo porrmmed cell-cell fusion assays on L.2 cell monolayers (see Materjals and Methods). By $24 \mathrm{~h}$ p.i, all of the recombinant viruses had induced syncytia formation, but at early times postinfection, there were clearly differences among the recombinanl viruses witli respect to their ability to induce cell-cell fusion. The percent fusion relative to $S_{4} R 29$ was found to be similar at 7 and $9 \mathrm{~h} \mathrm{p.i.;} \mathrm{the} \mathrm{results}$ at 7 hours are shown in Figure 5. Similar levels of cell-cell fusion were observed in $L 2$ cells infected with the (wo parontal recombinants, $S_{4} R$ and $S_{A 59} R$, and the severely attenuated recombinant $S 2_{4} R$, expressing the Si of MHV-A59 and the S2 of MHV4. In contrasi, recombinants expressing spikes with alterations in the I-IVR, $S_{4} \triangle H V R, S_{4} H V-\Lambda 59 R$, and $S_{A 59} H V-4 R$, or with pairing of the $S 1$ subunit of $M H V-$ 4 with the $S 2$ subunit of $M H V-A 59, S 1_{4} R$, all exhibited some clegree of delay in cell-cell fusion. Deletion of the MHV-4 IVR resulted in the most dramatic decrease in fusion, while replacement of the MHV-A59 HVR with the MHV-4 HVR resulted in anly a moderate decrouse in lusion. The delayed fusion formation observed with $S_{4} \triangle H V R$ is consistent with the observation by Gallagher et al (1990) that deletions in the HVR can result in decreased fusion. Thus, the neuroatenuated virulence observed with some but

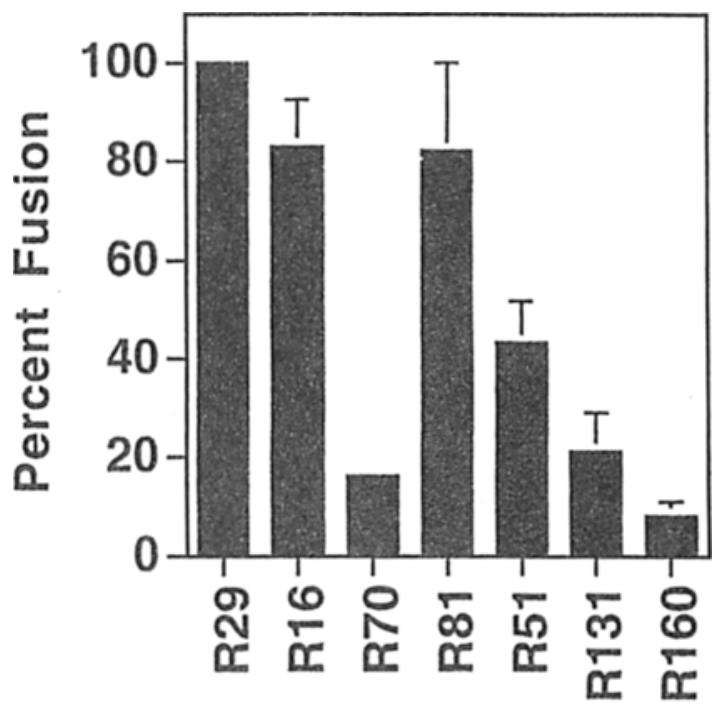

\section{Virus}

Figure 5 (A) Cell-cell fusion assay on L2 cell monolayers. Cells wore infected at a multiplicity of infection of 0.1 or 0.7 PFU/cell with $S_{i} R 29, S_{A 59} R 16, S 1_{i} R 70, S 2{ }_{4} R 81, S_{A 59} H V-4 R 51$, $S_{i}$ HV-A59R131, and $S_{4} \triangle H V R 160$. At $7 \mathrm{~h}$ p.i., cells were fixed. Using phase-contrast microscopy and a $20 \times$-objective the total number of nuclei in syncytia per field was determined for at least 10 random fied ds per 6-well plate. The percent fusion relative to that observed with $S_{i} R 29$ was calculated for each experiment and the means (and standard deviations) are shown. 
not all of the recombinants may be associated with decreased cell-cell fusion in cell culture.

\section{Discussion}

With the development of targeted recombination the structural genes of MHV have become amenable to genetic manipulation. Using this technique we previously demonstrated that the spike gene of the highly neurovirulent MHV-4 virus can confer a dramatic increase in virulence to the much less virulent MHVA59 virus (Phillips et al, 1999). Here we report on studies designed to localize this phenotype to a specific region of the spike, and to examine the effect of specific alterations in the spike on pathogenesis.

Using targeted recombination, we generated a series of recombinants containing MHV-4/MHV-A59 chimeric spike genes. We first characterized their in vitro phenotype to ensure that the chimeric spike genes were functional for infection and replication in tissue culture. We then examined the in vivo phenotype of the recombinants. The two parental recombinants, $S_{4} R$ (containing the MHV-4 spike gene) and $S_{A 59} R$ (containing the MHV-A59 spike gene), exhibit similar kinetics of replication in cell culture, but $S_{A 59} R$ achieves higher peak titers than $S_{4} R$. In cell culture, all of the chimeric spike gene recombinants replicated efficiently. They exhibited similar kinetics of replication as $S_{4} R$ and $S_{A 59} R$, and their peak titers were similar to or intermediate between the high titers observed with $S_{A 59} R$ and the lower titers observed with $\mathrm{S}_{4} \mathrm{R}$.

When we infected mice with the recombinant viruses, however, we observed that many of the recombinants displayed a different phenotype from either of the two parental recombinants. Recombinants containing exchanges between the S1 and S2 subunits $\left(S 1_{4} R\right.$ or $\left.S 2_{4} R\right)$ or deletion or replacement of the MHV-4 spike hypervariable region $\left(S_{4} \Delta H V R\right.$ or $\mathrm{S}_{4} \mathrm{HV}-\mathrm{A} 59 \mathrm{R}$, respectively) exhibited a profound decrease in viral replication in the brain as demonstrated by the titers of infectious virus. Furthermore, these recombinants were highly attenuated for neurovirulence as demonstrated by their high intracranial $L D_{50}$ values. The high titers of infectious virus in the brains of mice infected with recombinant $S_{\mathrm{A} 59} \mathrm{HV}$ $4 \mathrm{R}$, containing the MHV-A59 spike with the MHV-4 spike HVR, demonstrated that not all of the chimeric spike gene recombinants exhibited attenuated replication. In addition, the virulence of $S_{A 59} H V-4 R$ was not attenuated as it was similar to the parental $S_{A 59} R$ recgmbinant. As illustrated by the two parental recombinants decreased neurovirulence is not always associated with decreased titers of infectious virus in the brain. $S_{4} R$ and $S_{A 59} R$ exhibit a $3 \log _{10}$ difference in intracranial $L D_{50}$, yet when mice are infected with the same dose of virus, $S_{4} R$ and $S_{A 59} R$ exhibit similar titers of infectious virus in the brain. The chimeric spike gene recombinants, however, demonstrate that alterations in both $S 1$ and $S 2$ can result in reduced viral titers in the brain, and that this decrease in viral replication in the brain is associated with decreased neurovirulence.

The neuroattenuated phenotype of recombinants containing S1/S2 exchanges between the MHV-4 and MHV-A59 S genes suggest that under the stringent conditions of an in vivo infection homotypic S1/S2 interactions are required for efficient replication in the CNS. Moreover, in combination with previous studies associating mutations in $S 1$ and $S 2$ with neuroattenuation, these data suggest that regions within both subunits of the MHV-4 spike are required to confer the highly neurovirulent phenotype (Parker et al, 1989; Wang et al, 1992; Grosse and Siddell, 1994; Saeki et al, 1997). These regions of spike, which when mutated, deleted, or replaced influence neuropathogenesis, may interact structurally in the tertiary conformation of the protein, or cooperate functionally for efficient functioning of spike. There is experimental evidence to support the idea that regions of S1 and S2 closely interact. Variant viruses have been identified in which mutations in S1, responsible for receptor binding, alter fusion, and mutations in $\mathrm{S} 2$, responsible for viral fusion, alter the conformation of $\mathrm{S} 1$ or confer resistance to neutralization by soluble receptor (Gallagher et al, 1990; Grosse and Siddell, 1994; Saeki et al, 1997). Although the spike proteins of MHV4 and MHV-A59 share a high degree of amino acid homology, the severely attenuated phenotype of the recombinants with chimeric spike genes indicate that there are critical differences between the two spike genes in both S1 and S2. As has been demonstrated with Theiler's virus (Pritchard et al, 1993; Zhang et al, 1993), the in vivo attenuation observed with viruses containing chimeric viral proteins between similar strains demonstrates the complexity and sequence specificity of viral protein interactions, and attests to the stringent constraints imposed on a virus in vivo.

Additional recombinants containing alterations exclusively in the MHV-4 hypervariable region (HVR) demonstrated that this region of S1 is required for the high neurovirulence of the MHV-4 spike. Deletion or replacement of the MHV-4 HVR resulted in neuroattenuation of the recombinant viruses. These results definitively confirm the data from previous studies suggesting an association between mutations or deletions in the MHV-4 HVR and neuroattenuation (Dalziel et al, 1986; Fleming et al, 1986; Wege et al, 1988; Parker et al, 1989; Taguchi and Fleming, 1989; Gallagher et al, 1990; Wang et al, 1992). Although no single function has been attributed to the HVR, studies of variant viruses suggest that alterations in this region can influence S1-S2 interactions and fusion (Gallagher et al, 1990; Gallagher, 1997). In addition, the MHV-4 spike HVR contains neutralizing antibody epitopes and T-cell epitopes (Dalziel et al, 
1986; Taguchi and Fleming, 1989; Gallagher et al, 1990; Castro and Perlman, 1995), and thus alterations in this region could also influence the immune response to infection. Due to the severely attenuated in vivo replication and virulence exhibited by recombinants with deletion or replacement of the MHV4 spike HVR, however, it appears that this region is critical for the in vivo functioning of the MHV-4 spike.

In contrast, replacement of the MHV-A59 spike HVR with the corresponding region from the MHV-4 spike demonstrated that the HVR of MHV-4 was not sufficient to confer an increase in neurovirulence. Interestingly, the presence of the MHV-4 HVR in the MHV-A59 spike did not appear to alter the $L_{50}$ of the resulting recombinant virus. Thus, not only could the MHV-4 HVR grossly substitute for the corresponding region of MHV-A59, but the MHV-A59 spike HVR was able to tolerate an insertion of $\mathbf{5 2}$ amino acids with no obvious effect on neurovirulence.

To identify more subtle alterations or defects in the MHV-4/MHV-A59 chimeric spike proteins we tested a number of their in vitro properties. Previous studies have suggested that the fusogenicity of the MHV spike is an important factor in both viral spread in the host and in virus-induced cytotoxicity (Gallagher et al, 1990; Gallagher et al, 1992; Rao and Gallagher, 1998). To determine if the spike proteins of the attenuated recombinants exhibited an altered ability to induce cell-cell fusion we performed cell-cell fusion assays. Four of the recombinants $\left(\mathrm{S}_{4} \mathrm{R} 70, \mathrm{~S}_{4} \Delta \mathrm{HVR} 160\right.$, $\mathrm{S}_{4} \mathrm{HV}-\mathrm{A} 59 \mathrm{R} 131$, and $\mathrm{S}_{\mathrm{A59}} \mathrm{HV}$-4R51) exhibited some degree of delayed fusion. Although not all of the attenuated recombinants exhibited decreased cell-cell fusion, it appeared that a severe reduction in cellcell fusion was correlated with neuroattenuation. Interestingly, the slight decrease in cell-cell fusion observed with the recombinants containing a replacement of the MHV-A59 HVR with the MHV-4 HVR did not result in a corresponding decrease in virulence. This result suggests that MHV infection and replication in the CNS may require a certain threshold level of fusion. Although not all viruses with fusion defects exhibit neuroattenuation (ST Hingley, unpublished data), decreased fusogenicity of the MHV spike has previously been found to associate with decreased spread in the CNS (Gallagher et al, 1990; Fazakerley et al, 1992). Thus for some of the recombinant viruses decreased fusion resulting in decreased spread and dissemination may contribute to the decreased replication observed in the CNS.

In additian to cell-cell fusion we also examined the temperature sensitivity and the thermostability of the recombinant viruses. The temperature sensitivity of the recombinants, as determined by the plating efficiency at $39.5^{\circ} \mathrm{C}$ compared to at $37.0^{\circ} \mathrm{C}$, revealed no differences between the highly virulent and highly attenuated recombinant viruses (data not shown). The thermostability of the recombinant viruses was also examined by determining the decrease in viral stock titers over time at $40^{\circ} \mathrm{C}, \mathrm{pH} 7.3$ (data not shown). The neuroattenuated recombinants fell into a range of thermostabilities, however, they were bounded by the highly thermostabile $S_{A 59} R 16$ and much less thermostabile $\mathrm{S}_{4} \mathrm{R} 29$ (data not shown). Thus, there was no clear correlation between thermostability and neurovirulence.

Due to the many selective pressures inherent in an in vivo infection, small differences in the kinetics of an infection can dramatically affect pathogenesis. Such differences may explain why many of the recombinants with alterations in the spike displayed efficient replication in cell culture, yet highly inefficient replication in vivo. By creating recombinant viruses with heterotypic S1/S2 interactions we may have inadvertently altered either structural or functional interactions between the $\mathrm{S} 1$ and $\mathrm{S} 2$ subunits. These perturbations in spike may not have been apparent in cell culture due to differences between in vitro and in vivo infection such as receptor density on the host cell, the availability of free virus, the virion density required for an efficient infection, and the fusogenicity of the host cell. It is also possible that there were slight alterations in the processing or expression of the chimeric spike proteins that were not apparent in vitro. The lack of a correlation between replication in cell culture and replication in vivo is not unique to the chimeric spike gene recombinants as it has been observed previously for other MHV strains and for other viruses (Gallagher et al, 1990; Pritchard et al, 1993).

The targeted recombination technique is a powerful tool for studying MHV pathogenesis as defined alterations in spike can be introduced into isogenic viruses. As with all strains of MHV, point mutations can arise during viral replication. To decrease the impact of such secondary site mutations in the recombinant viruses we selected and characterized two independent recombinants for each desired recombinant, and sequenced the entire $S$ gene from at least one recombinant. Interestingly, $\mathrm{S}_{4} \mathrm{HV}$-A59R was the only recombinant for which multiple, independent recombinants were found to contain single coding mutations in $S$ after minimal passage in cell culture. The location of one of these compensatory mutations identified in $\mathrm{S} 2(\mathrm{L1114} F)$ is striking as numerous studies have identified alterations in this exact residue. The 1114 codon has been identified by Gallagher et al (1991) (L1114R) as one of three amino acids responsible for conferring $\mathrm{pH}$-dependent fusion on MHV-4, by Saeki et al (1997) (L1114F) in the spike gene of soluble-receptor resistant mutants, and by Wang et al (1992) (L1114F) in a neuroattenuated monoclonal antibody escape mutant. It is interesting to speculate that this region of S2 plays a prominent role in the conformation of spike and perhaps in the dynamic interaction that occurs between $S 1$ and $S 2$. The identification of compensatory mutations that 
arise both in vivo and in vitro as a result of various alterations in spike may provide valuable clues as to which regions of spike undergo functional and/or structural interactions.

The spike of MHV is a major determinant of neurovirulence. This is the first report using targeted recombination to examine the role of specific regions of the MHV spike in neuropathogenesis. Despite officient replication in cell culture many of the MHV4/MHV-A59 chimeric $S$ gene recombinants displayed severely attenuated replication in vivo. Thus, the degree of amino acid homology between the MHV-4 and MHV-A59 spike glycoproteins was high enough for the chimeric spike gene recombinants to replicate officiently in vitro, yet not bigh enough for efficient replication in the murine CNS. In addition, recombinants with alterations in the spike HVR have confirmed the importance of this region in the highly neurovirulent phenotype conferred by MHV-4 spike. Thus the pronounced neurovirulence conferred by the MHV-4 spike appears to depend on the structural and/or functional interaction of multiple regions of spike rather than on discreet independent functional domains within spike.

\section{Materials and methods}

Virus and cells

Feline FCWF cells, fMHV, and Alb4 were obtained from Paul S Masters (Albany, NY). The fMHV virus is a recombinant MHV that contains the ectodomain of the S protein of feline infectious peritonitis virus, the-Alb4 replicase, and the MHV-A59 $3^{\prime}$-end (Kuo et al, 2000). Alb4, derived from MHV-A59, is a temperature-sensitive $\mathrm{N}$ gene deletion mutant that produces small plaques at the non-permissive temperature $\left(39^{\circ} \mathrm{C}\right)$ and is thermolabile (Koetzner et al, 1992). Viruses were propagated on either murine 17Cl-1 cells or feline FCWF cells, and plaque assays and purifications were carried out on murine L2 cells. Cells were maintained on plastic tissue culture flasks in Dulbecco's minimal essential medium (MEM) with $10 \%$ fetal bovine serum (FBS). Spinner cultures of L2 cells were maintained in Joklik's MEM with $10 \%$ FBS at densities of between $2 \times 10^{5}$ and $2 \times 10^{6}$ cells per ml.

\section{Plasmids and PCR mutagenesis}

The pMH54 plasmid (obtained from Paul S Masters) includes from codon 28 of the $\mathrm{HE}$ pseudogene through to the $3^{t}$ end of the MHV-A59 genome as described by Kuo et al (2000). The pGEM4Z plasmid containing the MHV-4 S gene, pSwt, was obtained from Michael J Buchmeier (LaJolla, CA) (Gallagher et al, 1991). The pGEM-S4 and pGEM-A59 plasmids were derived from $\mathrm{pSwt}$ and contained the MHV-4 and the MHV-A59 spike genes, respectively (Phillips et al, 1999). These plasmids also contained silent mutations at codons 12 and 13 of the $S$ gene to create an
AvrII site, and at $12 \mathrm{nt}$ past the $\mathrm{S}$ gene stop codon to create a Sbfl site. These restriction sites were used for shuttling the various spike genes into the PMH54 vector.

To generate the various exchanges in thers gene, coding-silent changes were introduced into the MHV-4 and MHV-A59 spike genes in PGEM-S4 or pGEM-A59, respectively, that created unique and/or common restriction sites. DNA manipulations were carried out using standard methods (Maniatis et al, 1982). All cloning sites and regions generated by PCR mutagenesis were verified by sequencing, and the composition of each construct was checked by restriction analysis.

To exchange the MHV-4 and MHV-A59 S1 and S2 subunits an EcoRV site was introduced at codon 775 (MHV-4 sequence). AvrI/EcoRV or EcoRV/SbfI segments were then exchanged between the MHV-4 and MHV-A59 spikes. The resulting plasmids, pG-S1 containing the S1 of MHV-4 and the S2 of MHVA59, and $\mathrm{pG}-\mathrm{S} 24$, containing the $\mathrm{S} 1$ of MHV-A59 and the S2 of MHV-4, were digested with AvrI/SbfI and the chimeric $S$ genes were shuttled into the pMH54 plasmid creating pMH54-S14 and pMH54$\mathrm{S} 2{ }_{4}$. A S gene with the MHV-A59 spike and the MHV-4 HVR was generated by introducing silent mutations into the MHV-4 spike creating a Pstl site at codon 412 and a $\mathrm{Kpnl}$ site at codon 614. Using these two sites the resulting 606-nt MHV-4 fragment was cloned into the MHV-A59 spike creating plasmid pG-S ${ }_{\mathrm{A} 59} \mathrm{HV}-4$. The AvrII/Sbfl segment from this plasmid was then cloned into pMH54 creating plas mid pMH54-S ${ }_{A 59} \mathrm{HV}-4$. The MHV-A59 HVR was in troduced into the MHV-4 spike by introducing silent mutations in the MHV-A59 spike at codons 488 and 548 creating an EcoR47III site and a BglII site, respec tively. For cloning purposes a silent mutation creat ing a BglII site was also introduced into the MHV=4 $S$ gene at codon 600. Using the Eco47III and Bglin: restriction sites the MHV-A59 HVR was cloned into the corresponding region of the MHV-4 spike creat ing plasmid $\mathrm{pG}-\mathrm{S}_{4} \mathrm{HV}$-A59. The AvrII/SbfI segment from this plasmid was then cloned into $\mathrm{pMH54}$ cre ating pMH54-S 4 HV-A59. Deletion of the MHV-4 HVR was based on a previously identified deletion in the MHV spike (Dalziel et al, 1986; Parker et al, 1989); The 142-amino acid deletion between codons 434 and 575 and codon substitution at K433N was cres ated using PCR mutagenesis in pGEM-S4. The re sulting plasmid, $\mathrm{pG}-\mathrm{S}_{4} \Delta \mathrm{HV}$, was used to shuttle the AvrII/Sbfl region of $S$ into pMH54 creating pMH54a $\mathrm{S}_{4} \Delta \mathrm{HV}$.

For RNA transcription the designated pMH54 plas. mids were linearized just $3^{\prime}$ to the poly(A) tail by d: gestion with PacI.

\section{Targeted RNA recombination and selection} of recombinants

Targeted recombination was carried out, as described by Kuo (2000), between the recipient virus fMHV and 
synthetic capped RNAs transcribed from. pMH54-

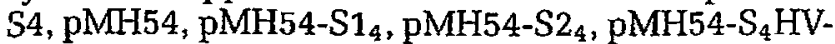
A59, and pMH54-S $4 \Delta$. Recombination with RNA transcribed from pMH54-S $\mathrm{Asg}_{\mathrm{A}} \mathrm{HV}-4$ was carried out with the Alb4 recipient virus as described previously (Masters et al, 1994; Leparc-Goffart et al, 1998; Phillips et al, 1999). The potential recombinants were plaque-purified and screened, using PCR amplification and gel electrophoresis, for the presence of the $5^{\prime}$ portion of the recombinant $S$ gene (Fischer et al, 1997; Leparc-Goffart et al, 1998; Phillips et al, 1999; Kuo et al, 2000). The selected recombinants were then plaque-purified once again, and viral stocks were grown on $17 \mathrm{Cl}-1$ cells for further analysis (Fischer et al, 1997). For each desired recombinant virus at least two recombinants derived from independent recombination events were characterized.

\section{$S$ gene sequencing}

For sequencing of the $S$ gene, cytoplasmic RNA extracted from virus-infected L2 cells was used as a template for reverse transcriptase-mediated PCR (RTPCR) amplification. The oligonucleotides described previously (Leparc-Goffart et al, 1998; Phillips et al, 1999) were used for amplification of the designated regions. Double-stranded PCR products were analyzed by automated sequencing by the Taq dye terminator procedure according to the manufacturer's protocol (Taq DyeDeoxy Terminator Cycle Sequencing kit; Applied Biosystems].

The entire $S$ gene was sequenced from at least one member of each pair of recombinant viruses. The sequence of each $S$ gene was identical to the previously published sequences for the MHV-4 and MHVA59 S genes (Luytjes et al, 1987; Parker et al, 1989; Gallagher et al, 1991; Phillips et al, 1999) except for recombinants containing the $\mathrm{S} 2$ of MHV-4, $S 2{ }_{4} \mathrm{R}$, and recombinants containing the MHV-4 spike with the MHV-A59 hypervariable region, $\mathrm{S}_{4} \mathrm{HV}-\mathrm{A} 59 \mathrm{R}$. S2 ${ }_{4} \mathrm{R} 81$ contained a single point mutation in the $S 1$ subunit that resulted in a codon change L661Q. S $2_{4} \mathrm{R} 82$, an independently isolated recombinant virus, contained no secondary site mutations in S. $S_{4} H V-A 59 R 131$ contained a single coding mutation S359I. An independently isolated recombinant, $\mathrm{S}_{4} \mathrm{HV}$-A59R133, also contained a single coding mutation N374Y. Two additional recombinants, $\mathrm{S}_{4} \mathrm{HV}-\mathrm{A} 59 \mathrm{R} 132$ and R134, were partially sequenced and found to contain single coding mutations (L1114F and L660Q, respectively).

\section{Virus growth curves}

Confluent L2 cell monolayers in 12-well plates were infected with each virus at the indicated multiplicity of infection. Infections were carried out in duplicate. Following adsorption for $1 \mathrm{~h}$ at $37^{\circ} \mathrm{C}$, the cells were washed with Tris-buffered saline three times and then fed with $1.5 \mathrm{~mL}$ of DMEM-10\% FBS. At the specified times, the cells were lysed by three cycles of freeze-thawing, and the supernatants were removed and titered by plaque assay on L 2 cells as previously described (Gombold et al, 1993).

\section{Animals}

$\mathrm{C} 57 \mathrm{Bl} / 6$ (B6) mice were purchased from the National Cancer Institute (Frederick, MD). Four-week-old, MHV-free male mice were used in all experiments. Mice were anesthetized with isoflurane (IsoFlo; Abbott Laboratories, North Chicago, IL). The amount of virus designated in each experiment was diluted in PBS containing $0.75 \%$ bovine serum albumin, and a total volume of $20 \mu \mathrm{l}$ was injected into the left cerebral hemisphere. Mock-infected controls were inoculated similarly but with an uninfected $17 \mathrm{Cl}-1$ cell lysate at a comparable dilution.

\section{Virulence assays}

Fifty percent lethal dose $\left(\mathrm{LD}_{50}\right)$ assays were carried out as described previously (Hingley et al, 1994). Mice were inoculated intracranially with 4- or 5-fold serial dilutions of recombinant viruses. A total of 5 animals per dilution per virus were analyzed. Mice were examined for signs of disease or death on a daily basis up to 21 days p.i. $L D_{50}$ values were calculated by the Reed-Muench method (Reed and Muench, 1938; Smith and Barthold, 1997).

\section{Infectious virus in the CNS}

The levels of infectious virus in the CNS as a function of time following infection with the recombinant viruses was determined in mice inoculated intracranially with $10 \mathrm{PFU}$ of virus. On days 1,3 , 5 , and 7 postinfection, mice were sacrificed, perfused with $10 \mathrm{ml}$ of PBS, and the brains were removed. The left-half of the brain was placed directly into $2 \mathrm{ml}$ of isotonic saline with $0.167 \%$ gelatin (gel saline) (Ramig, 1982). All organs were weighed and stored frozen at $-80^{\circ} \mathrm{C}$ until titered for virus. Brains were homogenized, and virus titers were determined by plaque-assay on L2 cell monolayers (Hingley et al, 1994). The titers of infectious virus in the brain were determined for all of the recombinant viruses at once in three independent experiments.

\section{Fusion assay}

To quantitate cell-cell fusion induced by the recombinant viruses, L2 cell monolayers in 6-well plates were infected with virus in duplicate (MOI 0.1 or 0.7 $\mathrm{PFU} / \mathrm{cell}$ ) and incubated for $1 \mathrm{~h}$ at $37^{\circ} \mathrm{C}$. Following adsorption, the cells were washed with Tris-buffered saline twice and then fed with $3 \mathrm{ml}$ of DMEM-10\% FBS. At 7 and $9 \mathrm{~h}$ p.i., the cells were washed with PBS and fixed with $2 \%$ paraformaldehyde (Sigma Chemical Co, St. Louis, MO). For each 20x-objective field under phase-contrast microscopy the total number of nuclei in syncytia was counted. At least 10 random fields were counted per well, and the mean 
number of nuclei in syncytia per field was calculated. The percent fusion relative to that observed with $S_{4} R 29$ was calculated for each experiment. To demonstrate that each $20 \times$-objective field had a similar number of cells, the total number of cells per field was counted at random. The results, shown in Figure 5, represent the means (and standard deviations) of at least three independent experiments except $S_{4} \triangle$ HVR160, which is from two independent experiments.

\section{References}

Banner LR, Keck JG, Lai MMC (1990). A clustering of RNA recombination sites adjacent to a hypervariable region of the peplomer gene of murine coronavirus. Virology 175: 548-555.

Beauchemin N, Draber P, Dveksler G, Gold P, Gray-Owen S, Grunert F, Hammarstrom S, Holmes KV, Karlsson A, Kuroki M, Lin S-H, Lucka L, Najjar SM, Neumaier M, Obrink B, Shively JE, Skubitz KM, Stanners CP, Thomas $P$, Thompson JA, Virji $M$, von Kleist $S$, Wagener $C$, Watt $S$, Zimmermann $W$ (1999). Redefined nomenclature for members of the carcinoembryonic antigen family. Exp Cell Res 252: 243-249.

Bergmann CC, Yao Q, Lin M, Stohlman SA (1996). The JHM strain of mouse hepatitis virus induces a spike proteinspecific $D^{b}$-restricted cytotoxic $T$ cell response. I Gen Virol 77: 315-325.

Buchmeier MJ, Lewicki HA, Talbot PJ, Knobler RL (1984). Murine hepatitis virus-4 (strain JHM)-induced neurologic disease is modulated in vivo by monoclonal antibody. Virology 132: 261-270.

Castro RF, Perlman S (1995). CD8+ T-cell epitopes within the surface glycoprotein of a neurotropic coronavirus and correlation with pathogenicity. / Virol 69: 81278131.

Collins AR, Knobler RL, Powell H, Buchmeier MJ (1982). Monoclonal antibodies to murine hepatitis virus-4 (strain JHM) define the viral glycoprotein responsible for attachment and cell-cell fusion. Virology 119: 358371.

Dalziel RG, Lampert PW, Talbot PJ, Buchmeier MJ (1986). Site-specific alteration of murine hepatitis virus type 4 peplomer glycoprotein E2 results in reduced neurovirulence. J Virol 59: 463-471.

DeGroot RJ, Luytjes W, Horzinek MC, van der Zeijst BAM, Spaan WJM, Lenstra JA (1987). Evidence for a coiledcoil structure in the spike proteins of coronaviruses. I Mol Biol 196: 963-966.

Dveksler GS, Pensiero MN, Cardellichio CB, Williams RK, Jiang G-S, Holmes KV, Dieffenbach CW (1991). Cloning of the mouse hepatitis virus (MHV) receptor: Expression in human and hampster cell lines confers susceptibility to MHV. J Virol 65: 6881-6891.

Fazakerley JK, Parker SE, Bloom F, Buchmeier MJ (1992). The V5A13.1 envelope glycoprotein deletion mutant of mouse hepatitis virus type-4 is neuroattenuated by its reduced rate of spread in the central nervous system. Virology 187: 178-188.

Fischer F, Stegen CF, Koetzner CA, Masters PS (1997). Analysis of a recombinant mouse hepatitis virus expressing a

\section{Acknowledgements}

This work was supported by Public Health Service grants NS-30606 and NS-21954. J.J.P. was supported in part by training grant GM-07229.

We thank Paul S Masters, Lili Kuo, and Peter JM Rottier for pMH54, Alb4, and fMHV. We thank Jean Tsai for providing us with $S_{A 5 B} R 16$, Danielle Linn Letting for her help, and Paul Bates, Amy Matthews, and Jean Tsai for critical reading of the manuscript.

foreign gene reveals a novel aspect of coronavirus transcription. J Virol 71: 5148-5160.

Fleming JO, Stohlman SA, Harmon RC, Lai MMC, Frelinger JA, Weiner LP (1983). Antigenic relationships of murine coronaviruses: Analysis using monoclonal antibodies to JHM (MHV-4) virus. Virology 131: 296-307.

Fleming JO, Trousdale MD, El-Zaatari FAK, Stohlman SA, Weiner LP (1986). Pathogenicity of antigenic variants of murine coronavirus JHM selected with monoclonal antibodies. J Virol 58: 869-875.

Frana MF, Behnke JN, Sturman LS, Holmes KV (1985). Proteolytic cleavage of the E2 glycoprotein of murine coronavirus: Host-dependent differences in proteolytic cleavage and cell fusion. I Virol 56: 912-920.

Gallagher TM (1997). A role for naturally occurring variation of the murine coronavirus spike protein in stabilizing association with the cellular receptor. $J$ Virol 71: 3129-3137.

Gallagher TM, Buchmeier MJ, Perlman S (1992). Cell receptor-independent infection by a neurotropic murine coronavirus. Virology 191: 517-522.

Gallagher TM, Escarmis C, Buchmeier MJ (1991). Alteration of $\mathrm{pH}$ dependence of coronavirus-induced cell fusion Effect of mutations in the spike glycoprotein. J Virol 65 1916-1928.

Gallagher TM, Parker SE, Buchmeier M] (1990) Neutralization-resistant variants of a neurotropic coro: navirus are generated by deletions within the aminoter minal half of the spike glycoprotein. / Virol 64: 731-741.

Gombold JL, Hingley ST, Weiss SR (1993). Fusion-defective mutants of mouse hepatitis virus $A 59$ contain a mutation in the spike protein cleavage signal. / Virol 67: 45044512.

Grosse B, Siddell SG (1994). Single amino acid changes in the S2 subunit of the MHV surface glycoprotein confer resistance to neutralization by $\mathrm{S} 1$ subunit-specific mon oclonal antibody. Virology 202: 814-824.

Hingley ST, Gombold JL, Lavi E, Weiss SR (1994). MHV A59 fusion mutants are attenuated and display altered hepatotropism. Virology 200: 1-10.

Koetzner CA, Parker MM, Ricard CS, Sturman LS, Masters PS (1992). Repair and mutagenesis of the genome of a deletion mutant of the murine coronavirus mouse hep atitis virus by targeted RNA recombination. J Virol 66 1841-1848.

Kubo H, Yamada YK, Taguchi F (1994). Localization of neu tralizing epitopes and the receptor-binding site within the amino-terminal 330 amino acids of the murine coro navirus spike protein. I Virol 68: 5404-5410. 
Kuo L, Godeke G-J, Raamsman MJB, Masters PS, Rottier PJM (2000). Retargeting of coronavirus by substitution of the spike glycoprotein ectodomain: Crossing the host cell species barrier. J Virol 74: 1393-1406.

Lavi E, Fishman PS, Highkin MK. Weiss SR (1988). Limbic encephalitis after inhalation of a murine coronavirus. Lab Investig 58: 31-36.

Lavi E, Gilden DH, Highkin MK, Weiss SR (1986). The organ tropism of mouse hepatitis virus strain A59 is dependent on dose and route of inoculation. Lab Anim Sci 36: 130135.

Leparc-Goffart I, Hingley ST, Chua MM, Phillips J, Lavi E, Weiss SR (1998). Targeted recombination within the spike gene of murine coronavirus mouse hepatitis virusA59: Q159 is a determinant of hepatotropism. J Virol 72: 9628-9636.

Luytjes W, Sturman LS, Bredenbeck P), Charite J, van der Zeijst BAM, Horzinek MC, Spaan WJM (1987). Primary structure of the glycoprotein E2 of coronavirus MHV-A59 and identification of the trypsin cleavage site. Virology 161: 479-487.

Manietis T, Fritsch EF, Sambrook J (1982). Molecular cloning, a laboratory manual. Cold Spring Harbor Laboratory: Cold Spring Harbor, New York.

Masters PS, Koetzner CA, Kerr CA, Heo Y (1994). Optimization of targeted RNA recombination and mapping of a novel nucleocapsid gene mutation in the coronavirus mouse hepatitis virus. J Virol 68: 328-337.

Parker SE, Gallagher TM, Buchmeier MJ (1989). Sequence analysis reveals extensive polymorphism and evidence of deletions within the E2 glycoprotein gene of several strains of murine hepatitis virus. Virology 173: 664673.

Pewe L, Wu GF, Barnett EM, Castro RF, Perlman S (1996). Cytotoxic $T$ cell-resistant variants are selected in a virus-induced demyelinating disease. Immunity 5: 253262:

Phillips J, Chua MM, Lavi E, Weiss SR (1999). Pathogenesis of chimeric MHV4/MHV-A59 recombinant viruses: The murine coronavirus spike protein is a major determinant of neurovirulence. I Virol 73: 7752-7760.

Pritchard AE, Jensen K, Lipton HI (1993). Assembly of Theiler's virus recombinants used in mapping determinants of neurovirulence. I Virol 67: 3901-3907.

Ramig RF (1982). Isolation and genetic characterization of temperature sensitive mutants of simian rotavirus SA11. Virology 120: 93-135.
Rao PV, Gallagher TM (1998). Intracellular complexes of viral spike and cellular receptor accumulate during cytopathic murine coronavirus infections. / Virol 72: 32783288.

Reed LJ, Muench $\mathrm{H}$ (1938). A simple method of estifhating fifty per cent points. Am J Hygeine 27: 493-497.

Saeki K, Ohtsuka N, Taguchi $F$ (1997). Identification of spike protein residues of murine coronavirus responsible for receptor-binding activity by use of soluble receptor-resisitant mutants. J Virol 71: 9024-9031.

Smith AL, Barthold SW (1997). Methods in viral pathogenesis. In Viral pathogenesis. Nathanson $\mathrm{N}$ (ed). Lippincott-Raven: Philadelphia, Pennsylvania, pp 483506.

Sturman LS, Ricard CS, Holmes KV (1990). Conformational change of the coronavirus peplomer glycoprotein at $\mathrm{pH}$ 8.0 and $37^{\circ} \mathrm{C}$ correlates with virus aggregation and virusinduced cell fusion. I Virol 64: 3042-3050.

Taguchi F, Fleming JO (1989). Comparison of six different murine coronavirus $\mathrm{JHM}$ variants by monoclonal antibodies against the E2 glycoprotein. Virology 169: 233235.

Tsai C-W, Chang SC, Chang M-F (1999), A 12-amino acid stretch in the hypervariable region of the spike protein S1 subunit is critical for cell fusion activity of mouse hepatitis virus. I Biol Chem 274: 26085-26090.

Wang F-I, Fleming JO, Lai MMC (1992). Sequence analysis of the spike protein gene of murine coronavirus variants: Study of genetic sites affecting neuropathogenicity. Virology 186: 742-749.

Wege $H$, Winter J, Meyermann $R$ (1988). The peplomer protein $\mathrm{E} 2$ of coronavirus JHM as a determinant of neurovirulence: Definition of critical epitopes by variant analysis. J Gen Virol 69: 87-98.

Weismiller DG, Sturman LS, Buchmeier MI, Fleming JO, Holmes KV (1990). Monoclonal antibodies to the peplomer glycoprotein of coronavirus mouse hepatitis virus identify two subunits and detect a conformational change in the subunit released under mild alkaline conditions. J Virol 64: 3051-3055.

Williams RK, Jiang GS, Holmes KV (1991). Receptor for mouse hepatitis virus is a member of the carcinoembryonic antigen family of glycoproteins. Proc Natl Acad Sci USA 88: 5533-5536.

Zhang L, Senkowski A, Shim B, Roos RP (1993). Chimeric cDNA studies of Theiler's murine encephalomyelitis virus neurovirulence. J Virol 67: 4404-4408. 